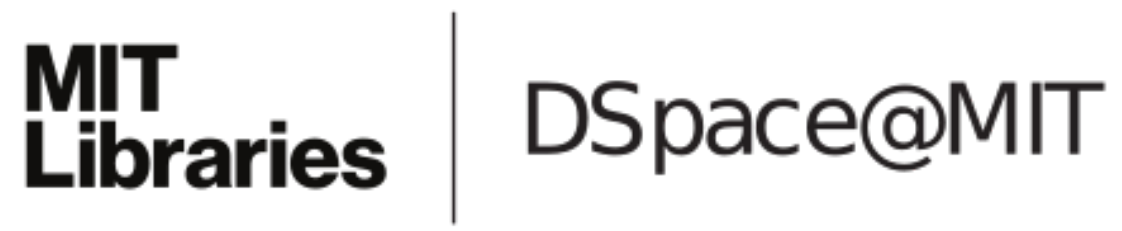

\author{
MIT Open Access Articles
}

Tensor renormalization group: Local magnetizations,
correlation functions, and phase diagrams
of systems with quenched randomness

The MIT Faculty has made this article openly available. Please share how this access benefits you. Your story matters.

Citation: Güven, Can, Michael Hinczewski, and A. Nihat Berker. “Tensor Renormalization Group: Local Magnetizations, Correlation Functions, and Phase Diagrams of Systems with Quenched Randomness." Physical Review E 82.5 (2010) : 051110. (c) 2010 The American Physical Society

As Published: http://dx.doi.org/10.1103/PhysRevE.82.051110

Publisher: American Physical Society

Persistent URL: http://hdl.handle.net/1721.1/63134

Version: Final published version: final published article, as it appeared in a journal, conference proceedings, or other formally published context

Terms of Use: Article is made available in accordance with the publisher's policy and may be subject to US copyright law. Please refer to the publisher's site for terms of use. 


\title{
Tensor renormalization group: Local magnetizations, correlation functions, and phase diagrams of systems with quenched randomness
}

\author{
Can Güven, ${ }^{1,2}$ Michael Hinczewski, ${ }^{3,4}$ and A. Nihat Berker ${ }^{5,6}$ \\ ${ }^{1}$ Department of Physics, University of Maryland, College Park, Maryland 20742, USA \\ ${ }^{2}$ Department of Physics, Koç University, Sarlyer, Istanbul 34450, Turkey \\ ${ }^{3}$ Feza Gürsey Research Institute, TÜBITAK-Bosphorus University, Çengelköy, Istanbul 34684, Turkey \\ ${ }^{4}$ Institute for Physical Science and Technology, University of Maryland, College Park, Maryland 20742, USA \\ ${ }^{5}$ Faculty of Engineering and Natural Sciences, Sabancı University, Orhanll-Tuzla, Istanbul 34956, Turkey \\ ${ }^{6}$ Department of Physics, Massachusetts Institute of Technology, Cambridge, Massachusetts 02139, USA
}

(Received 9 September 2010; published 8 November 2010)

\begin{abstract}
The tensor renormalization-group method, developed by Levin and Nave, brings systematic improvability to the position-space renormalization-group method and yields essentially exact results for phase diagrams and entire thermodynamic functions. The method, previously used on systems with no quenched randomness, is extended in this study to systems with quenched randomness. Local magnetizations and correlation functions as a function of spin separation are calculated as tensor products subject to renormalization-group transformation. Phase diagrams are extracted from the long-distance behavior of the correlation functions. The approach is illustrated with the quenched bond-diluted Ising model on the triangular lattice. An accurate phase diagram is obtained in temperature and bond-dilution probability for the entire temperature range down to the percolation threshold at zero temperature.
\end{abstract}

DOI: 10.1103/PhysRevE.82.051110

PACS number(s): 75.10.Nr, 05.10.Cc, 64.60.ah, 64.60.De

\section{INTRODUCTION}

The tensor renormalization-group (TRG) method developed by Levin and Nave [1] is a highly useful update of the traditional position-space renormalization-group approaches. While these founding approaches relied on uncontrolled approximations that were often system specific [2-7], the TRG is general in scope-it works on any classical twodimensional lattice Hamiltonian with local interactions - and its accuracy can be systematically improved to converge on the exact thermodynamic results. Along with these advantages, the method fits within the conceptual framework of traditional renormalization-group theory: it is a mapping between Hamiltonians on the original and coarse-grained lattices, and phase-transition behavior can be extracted from flows of the Hamiltonians as the transformation is iterated [8].

The initial TRG study demonstrated the power of the approach in the context of the triangular-lattice Ising model [1]. Since then it has proven a versatile tool for a variety of classical systems, including the frustrated Ising model on a Shastry-Sutherland lattice [9], relevant to magnetization plateaus in rare-earth tetraborides, and the zero-hopping limit of a model for ultracold bosonic polar molecules on a hexagonal optical lattice [10]. Moreover, the ideas behind the TRG method have become the kernel for developments in twodimensional quantum systems [11-16], most notably tensorentanglement renormalization group for studying symmetry breaking and topological phase transitions [11] and accurate methods to calculate ground-state expectation values [12-14]. Beyond the precision of the method, a key factor spurring the growth of TRG applications in both classical and quantum cases is computational efficiency: the CPU cost of carrying out TRG scales linearly with lattice size [14].

Given these promising characteristics, TRG is a natural candidate for tackling models with quenched randomness - a field where extracting accurate phase diagram information is a significant challenge. The current study presents the example of TRG applied to such a system with frozen disorder, namely, the percolative system of the bond-diluted triangular-lattice Ising ferromagnet, yielding, as seen in Fig. 1 , a highly accurate global phase diagram down to zero temperature, where it connects with the percolation transition.

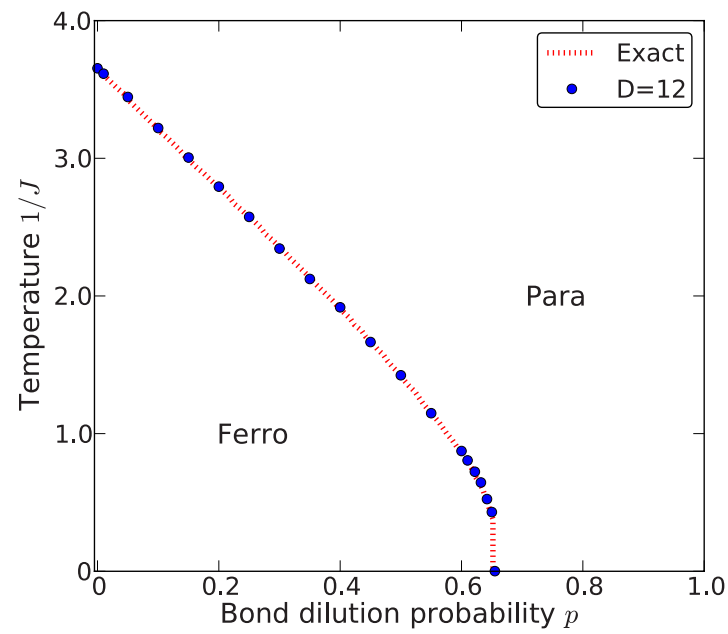

FIG. 1. (Color online) The phase diagram of the bond-diluted Ising model on a triangular lattice, showing the transition temperature as a function of the bond dilution probability $p$. The ferromagnetic (Ferro) and paramagnetic (Para) phases are marked. The phase boundary line between these two phases connects, at zero temperature, with the percolation transition on the triangular lattice. Filled circles are our results using the TRG method with $D=12$ together with finite-size scaling, as described in Sec. IV. The red dotted line is the result of the work of Georges et al. [17], which is exact on the scale of the figure. 


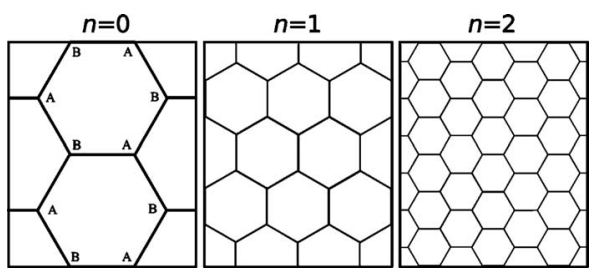

FIG. 2. Construction of the hexagonal lattice used in the TRG procedure. Starting from the initial structure on the left $(n=0)$, at each construction step, every vertex is replaced by a hexagon. Periodic boundary conditions are imposed between the top and bottom edges and between the left and right edges as if the lattice is on the surface of a torus. The sublattices $A$ and $B$ are shown for the $n=0$ step.

Our paper is organized as follows. Section II develops the TRG method for a general quenched random system. Section III illustrates this tensor network mapping, in particular, for the bond-diluted model and shows how to extract physical observables such as spin-spin correlation functions. Section IV uses this method, together with finite-size scaling relations for the correlation functions, to derive our main result: the phase diagram in terms of temperature vs bond dilution probability. Close agreement with the known critical temperature curve [17] is achieved even at a relatively low order of the TRG approximation (i.e., a small cutoff parameter). Our work opens up future possibilities for the extensive use of TRG in quenched disordered systems, as argued in the concluding remarks of Sec. V.

\section{TRG METHOD FOR QUENCHED RANDOM SYSTEMS}

\section{A. Tensor network}

As in earlier studies [1,8], we focus here on classical Hamiltonians associated with hexagonal-lattice tensor networks, though the method that we develop for quenched random systems is readily generalized to other geometries such as the square and kagome lattices [1]. We consider a general Hamiltonian that involves local interactions expressed in terms of bond degrees of freedom, such that each bond has $d$ possible states and the partition function of the system has the form

$$
Z=\sum_{i_{1}, \ldots, i_{K}=1}^{d} T_{i_{1} i_{2} i_{3}} T_{i_{3} i_{4} i_{5}} \ldots T_{i_{K-2} i_{K-1} i_{K}},
$$

where, for each of the $N$ sites in the hexagonal lattice, a real-valued tensor $T_{i_{m} i_{n} i_{o}}$ is a Boltzmann weight, depending on the configuration of the three bonds meeting at the site. The bond degrees of freedom correspond to each tensor index running from 1 to $d$. These bond indices are labeled $i_{1}$ through $i_{K}$ for the total of $K=3 N / 2$ bonds in the lattice. Although the tensor can have as many as $d^{3}$ distinct nonzero elements, in practice some bond configurations may be disallowed for a given Hamiltonian, corresponding to zerovalued tensor elements.

To facilitate the description of the TRG procedure, the hexagonal lattice is constructed as illustrated in Fig. 2: at the $n$th step, we replace each vertex with a hexagon, with the initial structure denoted $n=0$. We impose periodic boundary conditions, such that the top and bottom edges are equivalent, as well as the left and right edges, so that the lattice effectively lies on the surface of a torus. After the $n$th step, the system has $N=8 \times 3^{n}$ sites and $K=4 \times 3^{n+1}$ bonds. The TRG method involves a renormalization-group transformation that reverses this construction process, mapping the system at step $n$ to one at step $n-1$.

The hexagonal lattice of any size can be decomposed into two sublattices $A$ and $B$, such that the nearest neighbors of one type belong to the other type. As an example, we label the sublattices in the $n=0$ panel of Fig. 2. We distinguish the sublattice tensors with superscripts, $T_{i_{m} i_{n} i_{o}}^{A}$ or $T_{i_{m} i_{n} i_{o}}^{B}$. In the partition function sum of Eq. (1), each bond index $i_{m}$ appears twice, once within an $A$ tensor and once within the neighboring $B$ tensor linked through that bond. Thus, evaluating $Z$ consists of performing $K$ tensor contractions.

In addition to the bond variables, the general system we consider has quenched random degrees of freedom, though for notational simplicity we shall not explicitly show the dependence of $T$ on these. Physical observables $Q$ will be expressed as $[\langle Q\rangle]$, where $\langle\cdot\rangle$ denotes the thermodynamic average over the bond degrees of freedom and $[\cdot]$ denotes the configurational average over the quenched disorder.

\section{B. TRG transformation}

The TRG transformation consists of two steps known as rewiring and decimation. In the rewiring step, the bonds of every pair of neighboring tensors $T^{A}$ and $T^{B}$ are reconnected, rewriting them as a contraction of two new tensors $S^{A}$ and $S^{B}$. The reconnection pattern is illustrated in Figs. 3(a) and 3(b) and can be broken down into three basic cases (highlighted in different colors) involving different orientations of the initial $T^{A}$ and $T^{B}$ tensors. In our graphical convention, the vertex where three solid lines meet is a $T$ tensor and the vertex where three dashed lines meet is an $S$ tensor. Indices on a tensor, i.e., $T_{i j k}^{A}$, correspond to bonds labeled $i, j$, and $k$ arranged counterclockwise around the tensor, with the first index marking the vertical bond for the $T$ tensors and the horizontal bond for the $S$ tensors. Thus, for example, the three rewirings shown in Fig. 3(b) denote the mathematical identities,

$$
\begin{aligned}
& \text { Case 1: } \sum_{k=1}^{d} T_{m k l}^{A} T_{j k i}^{B}=\sum_{\nu=1}^{d^{2}} S_{l \nu j}^{A} S_{i \nu m}^{B}, \\
& \text { Case 2: } \sum_{k=1}^{d} T_{k l m}^{A} T_{k i j}^{B}=\sum_{\nu=1}^{d^{2}} S_{\nu j l}^{A} S_{\nu m i}^{B}, \\
& \text { Case 3: } \sum_{k=1}^{d} T_{l m k}^{A} T_{i j k}^{B}=\sum_{\nu=1}^{d^{2}} S_{j l \nu}^{A} S_{m i \nu}^{B} .
\end{aligned}
$$

Note that the $S$ tensors have two indices which run up to $d$ (labeled by Latin letters) and one index that runs up to $d^{2}$ (labeled by a Greek letter). The reason why $S^{A}$ and $S^{B}$ must 

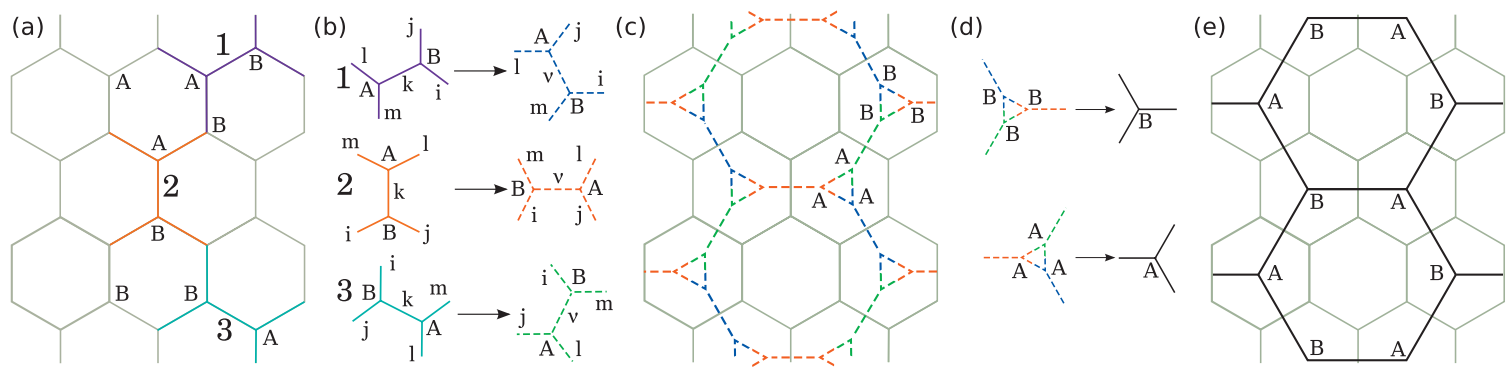

FIG. 3. (Color) The TRG transformation described in Sec. II B. (a) The hexagonal tensor network, with the three representative orientations of $T^{A}$ and $T^{B}$ tensor pairs, labeled as cases 1-3 and highlighted in different colors. (b) For each of the three cases, the rewiring step [Eq. (2)] expressing the contraction equivalently in terms of different tensors $S^{A}$ and $S^{B}$. (c) After every pair of tensors is rewired, the resulting martini lattice of $S^{A}$ and $S^{B}$ tensors. The original lattice is superimposed in gray for reference. (d) The decimation step [Eq. (3)], which replaces three $S^{A}$ tensors by a renormalized $T^{\prime A}$ tensor (and analogously for $S^{B}$ ). (e) The final lattice of renormalized $T^{\prime A}$ and $T^{\prime B}$ tensors, with the original lattice in gray for comparison.

have this structure comes from the following derivation, which also illustrates how one can explicitly calculate these tensors.

We shall describe the derivation for case 1 since the other two cases are analogous. The first line of Eq. (2) can be expressed as a $d^{2} \times d^{2}$ matrix equation, $M=S^{A}\left(S^{B}\right)^{T}$, where $M_{\alpha \beta} \equiv \Sigma_{k} T_{m k l}^{A} T_{j k l}^{B}, S_{\alpha \nu}^{A} \equiv S_{l \nu j}^{A}$, and $S_{\beta \nu}^{B} \equiv S_{i \nu m}^{B}$. Here, we use composite indices $\alpha$ and $\beta$ with $d^{2}$ states defined as $\alpha$ $\equiv(j, l)$ and $\beta \equiv(m, i)$. As a real-valued matrix, $M$ has a singular value decomposition of the form $M=U \Sigma V^{T}$, where $U$ and $V$ are orthogonal matrices and $\Sigma$ is a diagonal matrix containing the $d^{2}$ singular values of $M$. Once the singular value decomposition of $M$ is calculated, the elements of $S^{A}$ and $S^{B}$ are given by $S_{\alpha \nu}^{A}=\sqrt{\Sigma_{\nu \nu}} U_{\alpha \nu}$ and $S_{\beta \nu}^{B}=\sqrt{\Sigma_{\nu \nu}} V_{\beta \nu}$, where $\Sigma_{\nu \nu}$ is the $\nu$ th singular value, adopting the ordering convention from largest to smallest with increasing $\nu$.

After all $T^{A}$ and $T^{B}$ pairs are rewired, we have a so-called martini lattice of $S^{A}$ and $S^{B}$ tensors, shown in Fig. 3(c). The final step of the TRG transformation is decimation, which traces over the degrees of freedom in the triangles of the martini lattice, substituting for each triangle a renormalized tensor $T^{\prime A}$ or $T^{\prime B}$. Graphically, Fig. 3(d) shows the decimation of three $S^{A}$ tensors to form $T^{\prime A}$ and of three $S^{B}$ tensors to form $T^{\prime}$. The corresponding expressions in terms of tensor components are

$$
\begin{gathered}
\sum_{j, l, h=1}^{d} S_{\nu j l}^{A} S_{l \gamma h}^{A} S_{h j \delta}^{A}=T_{\nu \gamma \delta}^{\prime A}, \\
\sum_{m, i, h=1}^{d} S_{\nu m i}^{B} S_{i \gamma h}^{B} S_{h m \delta}^{B}=T_{\nu \gamma \delta}^{\prime B} .
\end{gathered}
$$

The final renormalized tensor network of $T^{\prime A}$ and $T^{\prime B}$ is shown in Fig. 3(e).

The partition function $Z$, a contraction over all bonds connecting the tensors [Eq. (1)], is exactly preserved through this transformation, as the hexagonal lattice is coarse grained from a step $n$ to a step $n-1$ structure. However, the indices of the renormalized tensors run from 1 to $d^{2}$ instead of 1 to $d$, so that if the TRG were iterated, arbitrarily large tensors would result, making numerical implementation difficult.
This problem is related to a general feature of position-space renormalization on lattices: except for specially tailored geometries (i.e., hierarchical lattices [18-20]), the number of couplings in the renormalized Hamiltonian grows with each coarse graining. For the TRG, we can tackle this issue in a systematic fashion by truncating the index range with an upper bound $D$. In Eq. (3) for $T^{\prime A}$ and $T^{\prime B}$, we shall allow the indices $\nu, \gamma$, and $\delta$ to run only up to $\bar{d} \equiv \min \left(d^{2}, D\right)$. This is equivalent to using truncated matrices $\bar{S}^{A}$ and $\bar{S}^{B}$ in the rewiring step, where $\bar{S}^{A}$ is the first $\bar{d}$ columns of the $d^{2} \times d^{2}$ matrix $S^{A}$ and $\bar{S}^{B}$ is the first $\bar{d}$ columns of $S^{B}$. As a result, the rewiring becomes approximate, $M \approx \bar{S}^{A}\left(\bar{S}^{B}\right)^{T}$. However, since the first $\bar{d}$ columns correspond to the largest singular values, the approximation is relatively accurate even for small $D$ and rapidly converges as $D$ is increased $[1,8]$. With this cutoff, the maximum size of the tensors is bounded as the TRG procedure is iterated, and we can extract numerically thermodynamic information from flows within a finite-dimensional space of real-valued tensor elements.

\section{TRG FOR QUENCHED RANDOMNESS: BOND- DILUTED ISING MODEL}

\section{A. Bond-diluted Ising Hamiltonian and its mapping onto a tensor network}

The general Hamiltonian for a quenched random Ising system is

$$
-\beta \mathcal{H}=\sum_{\langle i j\rangle}\left[J_{i j} s_{i} s_{j}+H_{i j}\left(s_{i}+s_{j}\right)\right], \quad s_{i}= \pm 1,
$$

where $\beta=1 / k_{B} T, J_{i j}$ and $H_{i j}$ are, respectively, the local spinspin coupling and magnetic field for sites $i$ and $j$, and $\langle i j\rangle$ denotes a sum over nearest-neighbor pairs of sites. Although this Hamiltonian encompasses a variety of models, all the way to the random-field spin glass [21], we shall here focus on a the bond-diluted Ising case, where the interaction constants $J_{i j}$ are distributed with a quenched probability $\mathcal{P}\left(J_{i j}\right)$ of the form 
type $A$

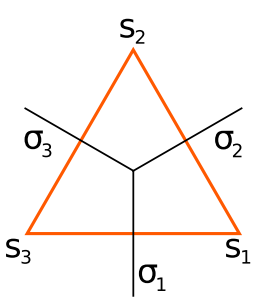

type $B$

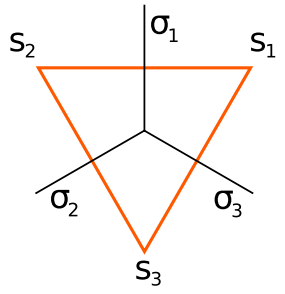

FIG. 4. (Color online) Duality mapping between spin states on the triangular lattice and bond variables in the tensor network. The variables $s_{i}= \pm 1$ at the triangle corners correspond to Ising spins in the Hamiltonian of Eq. (4). The bond variables $\sigma_{i}$ are products of the $s_{i}$ connected by the bond. Up and down triangles yield type A and B tensors, respectively.

$$
\mathcal{P}\left(J_{i j}\right)=p \delta\left(J_{i j}\right)+(1-p) \delta\left(J_{i j}-J\right) .
$$

Here, $J>0$, implying ferromagnetism and $p$ is the fraction of missing bonds. While we restrict our attention to the zero magnetic-field subspace, $H_{i j}=0$, formally the local fields will be kept in the Hamiltonian in order to take derivatives to obtain thermodynamic functions.

Starting with the Hamiltonian of Eq. (4) on a triangular lattice, a duality transformation allows us to express the partition function as a hexagonal-lattice tensor network. (The duality for Potts spins would generate three-point interactions, which would be included in the definition of the tensor $T_{i_{1} i_{2} i_{3}}$.) Each triangle in the triangular lattice corresponds to a tensor, with up triangles associated with a $T^{A}$ and down triangles with a $T^{B}$, as shown in Fig. 4. For spin variables $s_{i}, s_{j}$, and $s_{k}$ in a given triangle in the manner illustrated in the figure, we define corresponding edge variables $\sigma_{m}$ as the products of neighboring $s_{m}$; i.e., for the type $A$ triangle, $\sigma_{1}$ $=s_{3} s_{1}, \sigma_{2}=s_{1} s_{2}$, and $\sigma_{3}=s_{2} s_{3}$ and for the type B triangle, $\sigma_{1}=s_{1} s_{2}, \sigma_{2}=s_{2} s_{3}$, and $\sigma_{3}=s_{3} s_{1}$. Since $s_{m}= \pm 1$ and $\sigma_{m}$ $= \pm 1$, we can now introduce a composite index $i_{m} \equiv(5$ $\left.-\sigma_{m}-2 s_{m}\right) / 2$ which runs from 1 to 4 and describes the four possible states of the $m$ th triangle edge. Letting $J_{m}$ be the coupling $J_{i j}$ associated with this edge and $H_{m}=H_{i j}$ be the edge magnetic field, then the tensors for the two triangles types are

$$
\begin{aligned}
T_{i_{1} i_{2} i_{3}}^{A}= & \exp \left[\frac{1}{2}\left(\sum_{m=1}^{3} J_{m} \sigma_{m}+H_{m}\left(1+\sigma_{m}\right) s_{m}\right)\right] \\
& \times P\left(\sigma_{1} \sigma_{2} \sigma_{3}\right) \cdot P\left(\sigma_{1} s_{1} s_{3}\right) P\left(\sigma_{2} s_{2} s_{1}\right) P\left(\sigma_{3} s_{3} s_{2}\right), \\
T_{i_{1} i_{2} i_{3}}^{B}= & \exp \left[\frac{1}{2}\left(\sum_{m=1}^{3} J_{m} \sigma_{m}+H_{m}\left(1+\sigma_{m}\right) s_{m}\right)\right] \\
& \times P\left(\sigma_{1} \sigma_{2} \sigma_{3}\right) \cdot P\left(\sigma_{1} s_{1} s_{2}\right) P\left(\sigma_{2} s_{2} s_{3}\right) P\left(\sigma_{3} s_{3} s_{1}\right),
\end{aligned}
$$

where $P(x) \equiv(1+x) / 2$ is a projection operator. The $P$ factors in the tensors remove the bond states that do not correspond to a physically allowable spin configuration. As a result of the projection operators, only 8 out of the 64 elements in the tensor are nonzero. These are listed, for the first renormalization step, in the third and sixth columns of Table I for $T^{A}$ and $T^{B}$, respectively.

\section{B. Local magnetization and spin-spin correlation function}

In order to derive expressions for thermodynamic quantities in the tensor formalism, let us now restrict the notation $T^{A}$ and $T^{B}$ to tensors in the zero magnetic-field subspace. We place a local magnetic field $H_{k}$ only at a single location $k$. Let us call the two tensors which share this bond $\widetilde{T}^{A}$ and $\widetilde{T}^{B}$. These are the only two tensors in the system whose components are modified by the local field. The corresponding partition function is

$$
Z=\sum_{i_{1}, \ldots, i_{K}} T_{i_{1} i_{2} i_{3}}^{A} T_{i_{4} i_{5} i_{3}}^{B} \cdots \widetilde{T}_{i_{k} i_{l} i_{m}}^{A} \widetilde{T}_{i_{k} i_{n} i_{o}}^{B} \cdots T_{i_{K-2} i_{K-1} i_{K}}^{B} .
$$

Without loss of generality we take the contraction of the $\widetilde{T}^{A}$ and $\widetilde{T}^{B}$ tensors to be case 2 in Eq. (2) since the derivation

\begin{tabular}{|c|c|c|c|c|c|c|}
\hline \multirow{2}{*}{$\begin{array}{l}\text { Spin state } \\
\left(s_{1}, s_{2}, s_{3}\right)\end{array}$} & \multicolumn{3}{|c|}{ Type A } & \multicolumn{3}{|c|}{ Type B } \\
\hline & $\left(i_{1}, i_{2}, i_{3}\right)$ & $T_{i_{1} i_{2} i_{3}}^{A}$ & $D_{i_{1} i_{2} i_{3}}^{A}$ & $\left(i_{1}, i_{2}, i_{3}\right)$ & $T_{i_{1} i_{2} i_{3}}^{B}$ & $D_{i_{1} i_{2} i_{3}}^{B}$ \\
\hline$\uparrow \uparrow \uparrow$ & 111 & $e^{(1 / 2)\left(J_{1}+J_{2}+J_{3}+2 H_{1}+2 H_{2}+2 H_{3}\right)}$ & $e^{(1 / 2)\left(J_{1}+J_{2}+J_{3}\right)}$ & 111 & $e^{(1 / 2)\left(J_{1}+J_{2}+J_{3}+2 H_{1}+2 H_{2}+2 H_{3}\right)}$ & $e^{(1 / 2)\left(J_{1}+J_{2}+J_{3}\right)}$ \\
\hline$\uparrow \uparrow \downarrow$ & 214 & $e^{(1 / 2)\left(-J_{1}+J_{2}-J_{3}+2 H_{2}\right)}$ & 0 & 124 & $e^{(1 / 2)\left(J_{1}-J_{2}-J_{3}+2 H_{1}\right)}$ & $e^{(1 / 2)\left(J_{1}-J_{2}-J_{3}\right)}$ \\
\hline$\uparrow \downarrow \uparrow$ & 142 & $e^{(1 / 2)\left(J_{1}-J_{2}-J_{3}+2 H_{1}\right)}$ & $e^{(1 / 2)\left(J_{1}-J_{2}-J_{3}\right)}$ & 241 & $e^{(1 / 2)\left(-J_{1}-J_{2}+J_{3}+2 H_{3}\right)}$ & 0 \\
\hline$\uparrow \downarrow \downarrow$ & 243 & $e^{(1 / 2)\left(-J_{1}-J_{2}+J_{3}-2 H_{3}\right)}$ & 0 & 234 & $e^{(1 / 2)\left(-J_{1}+J_{2}-J_{3}-2 H_{2}\right)}$ & 0 \\
\hline$\downarrow \uparrow \uparrow$ & 421 & $e^{(1 / 2)\left(-J_{1}-J_{2}+J_{3}+2 H_{3}\right)}$ & 0 & 412 & $e^{(1 / 2)\left(-J_{1}+J_{2}-J_{3}+2 H_{2}\right)}$ & 0 \\
\hline$\downarrow \uparrow \downarrow$ & 324 & $e^{(1 / 2)\left(J_{1}-J_{2}-J_{3}-2 H_{1}\right)}$ & $-e^{(1 / 2)\left(J_{1}-J_{2}-J_{3}\right)}$ & 423 & $e^{(1 / 2)\left(-J_{1}-J_{2}+J_{3}-2 H_{3}\right)}$ & 0 \\
\hline$\downarrow \downarrow \uparrow$ & 432 & $e^{(1 / 2)\left(-J_{1}+J_{2}-J_{3}-2 H_{2}\right)}$ & 0 & 342 & $e^{(1 / 2)\left(J_{1}-J_{2}-J_{3}-2 H_{1}\right)}$ & $-e^{(1 / 2)\left(J_{1}-J_{2}-J_{3}\right)}$ \\
\hline$\downarrow \downarrow \downarrow$ & 333 & $e^{(1 / 2)\left(J_{1}+J_{2}+J_{3}-2 H_{1}-2 H_{2}-2 H_{3}\right)}$ & $-e^{(1 / 2)\left(J_{1}+J_{2}+J_{3}\right)}$ & 333 & $e^{(1 / 2)\left(J_{1}+J_{2}+J_{3}-2 H_{1}-2 H_{2}-2 H_{3}\right)}$ & $-e^{(1 / 2)\left(J_{1}+J_{2}+J_{3}\right)}$ \\
\hline
\end{tabular}
proceeds analogously for the other cases.

TABLE I. The tensor elements for the bond-diluted Ising model, as defined in Secs. III A and III B, for the first renormalization step. The first column gives the spin state $\left(s_{1}, s_{2}, s_{3}\right)$ for a triangle of the original triangular lattice, following the convention of Fig. 4 . For the type A triangle, the next three columns show the associated composite indices $\left(i_{1}, i_{2}, i_{3}\right)$ and the tensor elements $T_{i_{1} i_{2} i_{3}}^{A}$ and $D_{i_{1} i_{2} i_{3}}^{A}$. The last three columns show the analogous information for the type B triangle. All tensor elements not shown are zero. 


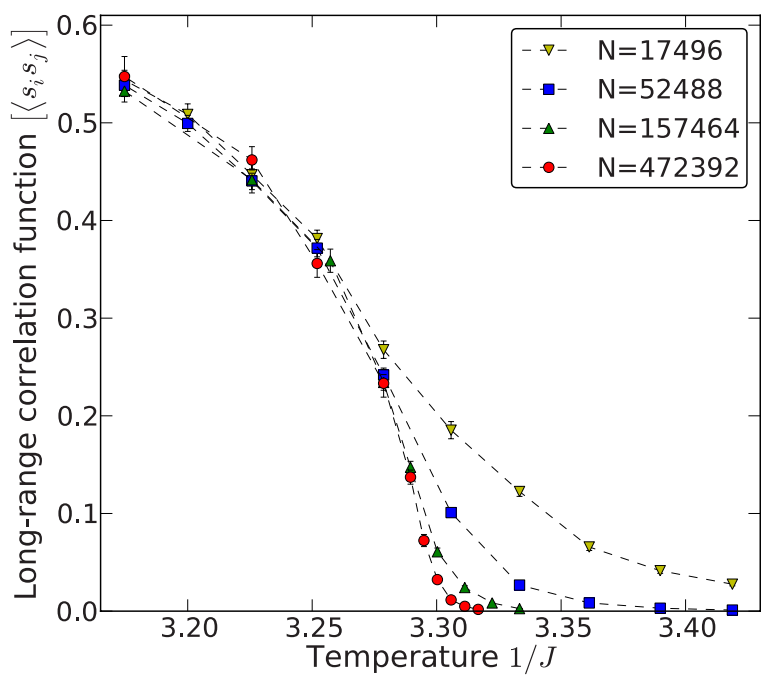

FIG. 5. (Color online) The long-distance spin-spin correlation $\left[\left\langle s_{i} s_{j}\right\rangle\right]$ as a function of temperature $1 / J$, calculated using the TRG method for bond dilution probability $p=0.1$ and cutoff parameter $D=8$. The curves for four different initial tensor network sizes $N$ are shown.

The local magnetization is $m_{k}=\left\langle\left(s_{i}+s_{j}\right) / 2\right\rangle \equiv\left\langle S_{k}\right\rangle$ for the sites $i$ and $j$ associated with the bond $k$. In terms of the local magnetic field $H_{k}$, the magnetization $m_{k}$ is given by the derivative

$$
\begin{aligned}
m_{k}=\left.\frac{1}{2} \frac{\partial \ln Z}{\partial H_{k}}\right|_{H_{k}=0}= & \frac{1}{2 Z} \sum_{i_{1}, \ldots, i_{K}}\left\{T_{i_{1} i_{2} i_{3}}^{A} T_{i_{4} i_{5} i_{3}}^{B} \cdots D_{i_{k} i_{l} i_{m}}^{A} T_{i_{k} i_{n} i_{o}}^{B} \cdots\right. \\
& \left.+T_{i_{1} i_{2} i_{3}}^{A} T_{i_{4} i_{5} i_{3}}^{B} \cdots T_{i_{k} i_{l} i_{m}}^{A} D_{i_{k} i_{n} i_{o}}^{B} \cdots\right\}
\end{aligned}
$$

where the differentiated tensors are

$$
D_{i_{k} i_{l} i_{m}}^{A}=\left.\frac{\partial \widetilde{T}_{i_{k} i_{l} i_{m}}^{A}}{\partial H_{i_{k}}}\right|_{H_{i_{k}}=0}, \quad D_{i_{k} i_{n} i_{o}}^{B}=\left.\frac{\partial \widetilde{T}_{i_{k} i_{n} i_{o}}^{B}}{\partial H_{i_{k}}}\right|_{H_{i_{k}}=0} .
$$

The nonzero elements of $D^{A}$ and $D^{B}$ are shown, for the first renormalization step, in the fourth and seventh columns of Table I.

After taking the average over the disorder, the first and second terms in the brackets on the right-hand side of Eq. (8) are equivalent, so that

$$
\left[m_{k}\right]=\left[\left\langle S_{k}\right\rangle\right]=\left[Z^{-1} \sum_{i_{1}, \ldots, i_{K}} T_{i_{1} i_{2} i_{3}}^{A} T_{i_{4} i_{5} i_{3}}^{B} \cdots D_{i_{k} i_{l} i_{m}}^{A} T_{i_{k} i_{n} i_{o}}^{B} \cdots\right] .
$$

A similar derivation for the correlation function yields

$$
\begin{aligned}
& {\left[\left\langle S_{k} S_{l}\right\rangle\right]} \\
& \quad=\left[Z^{-1} \sum_{i_{1}, \ldots, i_{K}} T_{i_{1} i_{2} i_{3}}^{A} T_{i_{4} i_{5} i_{3}}^{B} \cdots D_{i_{k} i_{l} i_{m}}^{A} T_{i_{k} i_{n} i_{o}}^{B} \cdots D_{i_{l} i_{p} i_{q}}^{A} T_{i_{l} i_{r} i_{s}}^{B} \cdots\right]
\end{aligned}
$$

We shall be interested in long-range correlations as an indicator of thermodynamic phase behavior. In this case, the four individual $s_{i}$ spin-spin correlations that make up the $\left[\left\langle S_{k} S_{l}\right\rangle\right]$

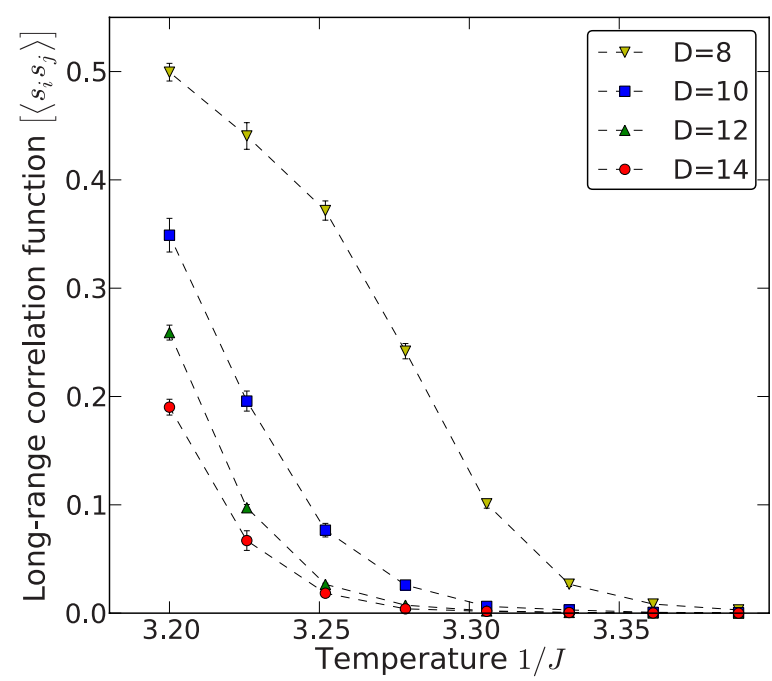

FIG. 6. (Color online) The long-distance spin-spin correlation $\left[\left\langle s_{i} s_{j}\right\rangle\right]$ as a function of temperature $1 / J$, calculated using the TRG method for bond dilution probability $p=0.1$ and network size $N$ $=157464$ tensors. The curves for four different cutoff parameters $D$ are shown.

are approximately equal: $\left[\left\langle S_{k} S_{l}\right\rangle\right] \approx\left[\left\langle s_{i} s_{j}\right\rangle\right]$, where $s_{i}$ is either of the spins contributing to $S_{k}$ and $s_{j}$ is either of the spins contributing to $S_{l}$. Hence, we shall use $\left[\left\langle S_{k} S_{l}\right\rangle\right]$ and $\left[\left\langle s_{i} s_{j}\right\rangle\right]$ interchangeably in the rest of the text.

\section{Details of numerical implementation}

To calculate the long-range spin-spin correlation function $\left[\left\langle S_{k} S_{l}\right\rangle\right]$, we start with a finite hexagonal lattice after $n$ construction steps, with size varying between $n=7-10$ steps ( $N=17$ 496-472 392 tensors). The bonds $k$ and $l$ are chosen to be at the maximum separation within the lattice, taking periodic boundary conditions into account. For a given realization of the disorder, the sum on the right-hand side of Eq. (11) is evaluated by doing $n$ TRG transformations, which yields the contraction in terms of four renormalized tensors in the $n=0$ structure. These last four tensors are directly contracted. A similar process yields the value of the partition function $Z$ which is the denominator in Eq. (11). The configurational average is taken over 200-300 realizations, implemented by randomly assigning the $J_{i j}$ on the initial lattice according to the probability distribution in Eq. (5). The tensors on the original lattice, i.e., in Eqs. (6) and (9), have index range $d=4$. For subsequent tensors, we use a cutoff parameter $D=8-14$.

Some tensor elements tend to grow exponentially in magnitude as the TRG transformation is iterated, which poses potential numerical difficulties. To counteract this, we take advantage of the fact that we can always factor out a constant from each tensor without changing the physics. For each tensor during each TRG iteration, the factor extracted is equal to $\min \left(T_{\max }, 2\right)$, where $T_{\max }$ is the maximum absolute value of the tensor elements. Keeping an upper bound of 2 on this extracted factor slows down the decay of most tensor elements to zero, which would otherwise lead to other numerical artifacts. We keep track of the total extracted factors 
in the numerator and denominator of Eq. (11), which are then used in calculating the final correlation function value.

\section{RESULTS}

Representative results for the long-distance spin-spin correlation function $\left[\left\langle s_{i} s_{j}\right\rangle\right]$ as a function of temperature $1 / J$ at bond dilution $p=0.1$ are given in Figs. 5 and 6. The former shows curves for various tensor network sizes $N$ using cutoff $D=8$, while the latter varies the cutoff $D$ at fixed size $N$ $=157$ 464. Away from the critical temperature, where widely separated spins are uncorrelated, $\left[\left\langle s_{i} s_{j}\right\rangle\right] \approx\left[\left\langle s_{i}\right\rangle^{2}\right]$, and we expect distinct limiting behaviors for the two different thermodynamic phases in the system: at low $1 / J$ in the ferromagnetically ordered phase $\left[\left\langle s_{i} s_{j}\right\rangle\right] \rightarrow 1$, while at high $1 / J$ in the paramagnetic phase $\left[\left\langle s_{i} s_{j}\right\rangle\right] \rightarrow 0$. The temperature region where one sees a smooth transition between these two regimes for finite systems, illustrated in Figs. 5 and 6, gives a rough indication of the phase-transition temperature $1 / J_{c}$ in the thermodynamic limit. With increasing $N$ in Fig. 5 and increasing $D$ in Fig. 6, the transition becomes sharper, as our truncations converge toward the exact result for an infinite system. The probability $p=0.1$ at which these results are calculated is smaller than the threshold value $p_{c} \approx 0.653$ [22], above which the triangular lattice no longer percolates. For $p>p_{c}$ we would not see a transition region: the paramagnetic phase exists at all temperatures since islands of ordered spins of size $\sim O(N)$ become exponentially improbable.

To obtain an accurate estimate of the exact transition temperature $1 / J_{c}$, we can employ the following finite-size scaling relation, which describes the ratios of the correlation functions at three different system sizes $N_{1}, N_{2}$, and $N_{3}$ when $J=J_{c}[23]$ :

$$
\frac{\ln \left(\frac{g\left(N_{2}\right)}{g\left(N_{1}\right)}\right)}{\ln \left(\frac{N_{2}}{N_{1}}\right)}=\frac{\ln \left(\frac{g\left(N_{3}\right)}{g\left(N_{2}\right)}\right)}{\ln \left(\frac{N_{3}}{N_{2}}\right)},
$$

where $g(N)$ is the long-distance correlation function $\left[\left\langle s_{i} s_{j}\right\rangle\right]$ for network size $N$. For the $i$ th system, at the temperature region where $g\left(N_{i}\right)$ decays rapidly to zero $(J$ just smaller than $J_{c}$ ), the decay is approximately exponential in $J$,

$$
\ln \left[g\left(N_{i}\right)\right] \approx A_{i} J-B_{i},
$$

for some constants $A_{i}$ and $B_{i}$. This exponential behavior for three different system sizes is shown in Fig. 7 for $p=0.25$ and 0.55 . To calculate $A_{i}$ and $B_{i}$, we do a weighted linear least-squares fit to $\ln \left[g\left(N_{i}\right)\right]$ vs $J$ data in a region of $J$ where the relative uncertainty (from the configurational average) for the data points is less than $15 \%$. Plugging Eq. (13) into Eq. (12) with $J=J_{c}$, we can solve for $J_{c}$ in terms of the $A_{i}, B_{i}$, and $N_{i}$ :

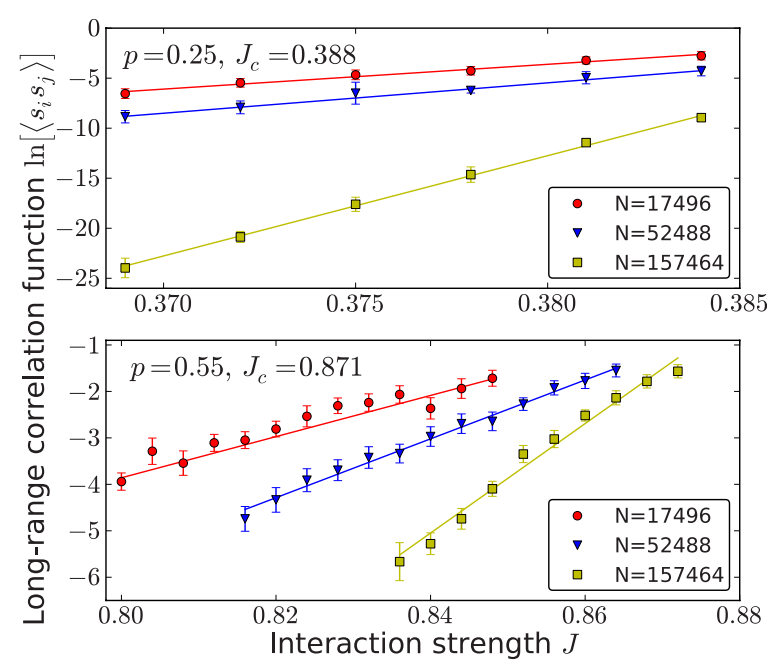

FIG. 7. (Color online) Data points show the logarithm of the long-distance spin-spin correlation, $\ln \left[\left\langle s_{i} s_{j}\right\rangle\right]$, as a function of interaction strength $J$ for three different system sizes $N$ and two different bond dilution probabilities $p$ (top panel: $p=0.25$; bottom panel: $p$ $=0.55$ ). The weighted least-squares linear fits, shown as solid lines, yield the coefficients $A_{i}$ and $B_{i}$ in Eq. (13), which allow one to estimate $J_{c}$ through finite-size scaling [Eq. (14)]. The resulting values of $J_{c}$ are $0.388(p=0.25)$ and $0.871(p=0.55)$.

$$
J_{c}=\frac{\left(B_{2}-B_{1}\right) \ln \left(\frac{N_{3}}{N_{2}}\right)+\left(B_{2}-B_{3}\right) \ln \left(\frac{N_{2}}{N_{1}}\right)}{\left(A_{1}-A_{2}\right) \ln \left(\frac{N_{3}}{N_{2}}\right)+\left(A_{3}-A_{2}\right) \ln \left(\frac{N_{2}}{N_{1}}\right)} .
$$

Carrying out this calculation across the entire $p$ range for $N_{1}=17496, N_{2}=52488$, and $N_{3}=157464$ at $D=12$, we obtain the phase diagram shown in Fig. 1. For comparison we also plot the same phase diagram obtained from a rigorous approximation scheme for the bond-diluted Ising-model free energy [17], which can be considered exact on the scale of the figure. The agreement is quite close, with an average relative deviation of $1 \%$. Two values along the curve are known exactly: $1 / J_{c}=4 / \ln 3=3.641[24]$ at $p=0$ and the curve goes to $1 / J_{c}=0$ at the percolation threshold $p=p_{c}=1$ $-2 \sin (\pi / 18)=0.653$ [22]. Our results deviate from these exact values by $0.3 \%$ and $0.4 \%$, respectively.

\section{CONCLUSIONS}

We have shown how the TRG approach provides an efficient and precise method for calculating thermodynamic properties of a quenched random classical model-the triangular-lattice bond-diluted Ising Hamiltonian. By expressing the partition function and related quantities such as spin-spin correlation functions in terms of tensor networks, they can be readily evaluated through TRG for large lattice sizes. In combination with finite-size scaling ideas, the result is a precise estimate of the phase diagram. If desired, convergence to the exact critical properties can be achieved by increasing the cutoff parameter defining the index range of the tensors. 
The bond-diluted Ising model is only a first step in the exploration of disordered systems using TRG: the methods presented here are easily extended to frustrated Hamiltonians exhibiting spin-glass behavior and the resulting complex multicritical phase structures. The numerical accuracy of the technique will be a valuable feature in probing analytical conjectures on the exact locations of spin-glass multicritical points [25-28].

\section{ACKNOWLEDGMENTS}

This research was supported by the Alexander von Humboldt Foundation, the Scientific and Technological Research Council of Turkey (TÜBITAK), and the Academy of Sciences of Turkey. Computational resources were provided by the Gilgamesh cluster of the Feza Gürsey Research Institute.
[1] M. Levin and C. P. Nave, Phys. Rev. Lett. 99, 120601 (2007).

[2] Th. Niemeijer and J. M. J. van Leeuwen, Phys. Rev. Lett. 31, 1411 (1973).

[3] A. A. Migdal, Zh. Eksp. Teor. Fiz. 69, 1457 (1975) [Sov. Phys. JETP 42, 743 (1976)].

[4] L. P. Kadanoff, Ann. Phys. (N.Y.) 100, 359 (1976).

[5] L. P. Kadanoff, Phys. Rev. Lett. 34, 1005 (1975).

[6] L. P. Kadanoff, A. Houghton, and M. C. Yalabık, J. Stat. Phys. 14, 171 (1976).

[7] A. N. Berker and M. Wortis, Phys. Rev. B 14, 4946 (1976).

[8] M. Hinczewski and A. N. Berker, Phys. Rev. E 77, 011104 (2008).

[9] M. C. Chang and M. F. Yang, Phys. Rev. B 79, 104411 (2009).

[10] L. Bonnes, H. Büchler, and S. Wessel, New J. Phys. 12, 053027 (2010).

[11] Z.-C. Gu, M. Levin, and X.-G. Wen, Phys. Rev. B 78, 205116 (2008).

[12] H. C. Jiang, Z. Y. Weng, and T. Xiang, Phys. Rev. Lett. 101, 090603 (2008).

[13] Z. Y. Xie, H. C. Jiang, Q. N. Chen, Z. Y. Weng, and T. Xiang, Phys. Rev. Lett. 103, 160601 (2009).

[14] H. H. Zhao, Z. Y. Xie, Q. N. Chen, Z. C. Wei, J. W. Cai, and T. Xiang, Phys. Rev. B 81, 174411 (2010).
[15] P. Chen, C.-Y. Lai, and M.-F. Yang, J. Stat. Mech.: Theory Exp. (2009), P10001.

[16] W. Li, S.-S. Gong, Y. Zhao, and G. Su, Phys. Rev. B 81, 184427 (2010)

[17] A. Georges, D. Hansel, P. Le Doussal, J. M. Maillard, and J. P. Bouchaud, J. Phys. France 47, 947 (1986).

[18] A. N. Berker and S. Ostlund, J. Phys. C 12, 4961 (1979).

[19] R. B. Griffiths and M. Kaufman, Phys. Rev. B 26, 5022 (1982).

[20] M. Kaufman and R. B. Griffiths, Phys. Rev. B 30, 244 (1984).

[21] G. Migliorini and A. N. Berker, Phys. Rev. B 57, 426 (1998).

[22] D. Stauffer and A. Aharony, Introduction to Percolation Theory (Taylor \& Francis, London, 1994).

[23] H. Takano and Y. Saito, Prog. Theor. Phys. 73, 1369 (1985).

[24] G. H. Wannier, Phys. Rev. 79, 357 (1950); Phys. Rev. B 7, 5017(E) (1973).

[25] H. Nishimori, J. Phys. Soc. Jpn. 71, 1198 (2002).

[26] M. Hinczewski and A. N. Berker, Phys. Rev. B 72, 144402 (2005).

[27] M. Ohzeki, H. Nishimori, and A. N. Berker, Phys. Rev. E 77, 061116 (2008).

[28] M. Ohzeki, Phys. Rev. E 79, 021129 (2009). 